\title{
Historical and interpretative aspects of quantum mechanics: a physicists' naive approach*
}

\author{
B.Berche, C.Chatelain, C.Dufour, T.Gourieux, D.Karevski \\ Groupe M, Laboratoire de Physique des Matériaux, UMR CNRS 7556, \\ Université Henri Poincaré, Nancy 1, F-54506 Vandœuvre les Nancy Cedex, France \\ Received April 10, 2006, in final form April 17, 2006 \\ Many theoretical predictions derived from quantum mechanics have been confirmed experimentally for the \\ last 80 years. However, interpretative aspects have long been subject to debate. Among them, the question \\ of the existence of hidden variables is still open. We review these questions, paying special attention to his- \\ torical aspects, and argue that one may definitively exclude local realism based on the present experimental \\ outcomes. Nevertheless other interpretations of Quantum Mechanics are not excluded.
}

Key words: foundations of quantum mechanics, EPR, entanglement, hidden variables, no-go theorems, Bell inequalities, Bohm theory

PACS: $01.65 .+g, 01.70 .+w, 03.65 . T a$

\section{Contents}

1 Motivations

2 1935: the reality assumptions in quantum mechanics

2.1 EPR assumptions . . . . . . . . . . . . . . . . . . . . . . . . . 321

2.2 Incompleteness of quantum mechanics . . . . . . . . . . . . . . . . . 321

2.3 Bohr's reply . . . . . . . . . . . . . . . . . . . . . . . 322

2.4 Bohm's further explanations . . . . . . . . . . . . . . . . . . . . . . 323

2.5 Einstein's point of view on separability . . . . . . . . . . . . . . . . . . . 324

2.6 What orthodox physicists should accept, and what others may reject . . . . . . . . 324

3 On the impossibility of local hidden variables $\quad 325$

3.1 Hidden-variable theories . . . . . . . . . . . . . . . . . . . . . . . . 325

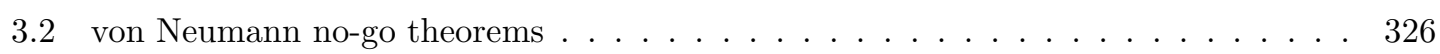

3.3 Bell inequalities . . . . . . . . . . . . . . . . . . . . . 327

3.4 A simple illustration of Bell's inequalities . . . . . . . . . . . . . . . 328

4 Tests of Bell's inequalities: from thought experiments to realistic experiments 328

4.1 Optical variant of the Bohm experiment . . . . . . . . . . . . . . . . . . . 329

4.2 Towards experimental tests: the $\mathrm{CHSH}$ inequalities . . . . . . . . . . . . . . 330

4.3 First experiments in the 70 's . . . . . . . . . . . . . . . . . . . . 331

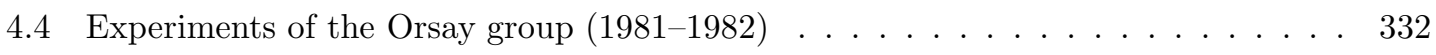

4.5 Toward the ideal experiment . . . . . . . . . . . . . . . . . . 333

${ }^{*}$ Talk given at the Mochima theoretical physics spring school, June 20th-24th 2005, by C. Dufour. 
5 Neo-Bohmian interpretation $\quad 334$

5.1 Orthodox interpretation, a few comments . . . . . . . . . . . . . . . 334

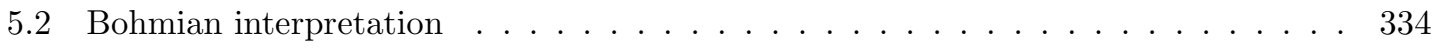

5.3 Criticism of the de Broglie-Bohm theory . . . . . . . . . . . . . . . . . 337

6 Discussion

\section{Motivations}

According to Mermin, contemporary physicists come in two varieties: those who are bothered by EPR arguments and Bell's theorem and those, perhaps the majority, who are not. In general, physicists who are not troubled by the kind of arguments used in the orthodox interpretation focus their attention on the technical part of quantum mechanics and on its unquestionable successes. This positive attitude leads most of the time to a kind of "operational realism" and to a complete disinterest regarding other interpretations. The present paper is a collection of lectures given at the Mochima summer school and at the university Henri Poincaré in Nancy. The aim of these lectures was to engage students and colleagues full of certitudes, in a discussion on some of the arguments bothering some contemporary physicists. It is a short, superficial and non-exhaustive review of the kind of questions that part of the community is dealing with.

In orthodox quantum mechanics a system is fully characterised by its wave function. However, in 1935 Einstein, Podolsky and Rosen (EPR) argued that such a theory is not complete, in the sense that not every element of reality has a counterpart in the theory. Bohr's reply underlined the fact that the EPR argumentation is misleading, since the interaction of the system with the experimental set-up is not taken into account. We discuss firstly the reality assumption in the celebrated debate between Einstein and Bohr.

If not convinced by Bohr's arguments, one may invoke additional parameters - the so-called hidden variables - in order to fully account for a system's properties. Historically, short after the EPR paper, the quest for hidden-variable theories weakened because the theorems of von Neumann, Gleason and Kochen as well as Specker claimed the impossibility of constructing hidden-variable theories reproducing all the results of quantum mechanics. In fact, these theorems have been shown to imply that such theories should exhibit contextuality (the dependence of a given measurement on the experimental set-up), rather than imply that hidden-variable interpretations are untenable. The subsequent step was Bell's assertion that any realistic local hidden-variable theory must satisfy inequalities that are violated by quantum mechanics. The theorem of von Neumann and the Bell inequalities are detailed in the third section.

The fourth section summarises the long series of experiments performed - mainly in quantum optics - since the beginning of the seventies and recently. Step-by-step improvements of the experimental expertise concerning the creation and manipulation of quantum states have led to the realization of several generations of experiments. These successive experimental tests, focusing essentially on the effort made to circumvent the locality loophole, favoured orthodox quantum mechanics and strongly indicates that local hidden-variable theories can be ruled out.

However the hidden-variable theories in general have not been disqualified. For example, the non-local Bohmian mechanics still holds. This theory, which can be considered as a successful construction of hidden-variable theory, is described in the fifth section. It is empirically equivalent to orthodox quantum theory.

We stress that we made no attempt at an exhaustive reference to the (vast) literature in the field. Interested readers may refer to recent reviews, e.g. [1].

\section{1935: the reality assumptions in quantum mechanics}

In a seminal paper published in 1935 [2], Einstein, Podolsky and Rosen (EPR) pointed out a tricky question about the epistemological interpretation of quantum mechanics. Their objection is supposed to prove the incompleteness of the theory with respect to physical reality. According to 
quantum mechanics, all the available physical information concerning a system is encoded in its wave function. If that was not the case, as argued by EPR in their paper, one would have to search for a more satisfying (complete) theory which would encompass quantum mechanics.

\subsection{EPR assumptions}

According to EPR, in a complete theory, "every element of the physical reality must have a counterpart in the physical theory". This condition requires an experimental specification in order to identify such an "element of the physical reality". Thus, EPR propose a sufficient criterion for their following argumentation: "if, without in any way disturbing a system, we can predict with certainty (i.e. with a probability equal to unity) the value of a physical quantity, then there exists an element of physical reality corresponding to this physical quantity".

Consider a particle in a momentum eigenstate, $\left|\mathbf{p}_{0}\right\rangle$ [i.e. with a wave function $\left.\psi \propto \exp \left(\operatorname{ip}_{0} \mathbf{r} / \hbar\right)\right]$. The momentum being $\mathbf{p}_{0}$ with certainty, it is then meaningful to consider that it is an element of the physical reality associated with the particle. On the other hand, the location of the particle is not determined in the state $\left|\mathbf{p}_{0}\right\rangle$, since all possible values of the coordinate are equally likely. Certainly, one may proceed to a direct measurement. But this measurement would perturb the state of the particle in such a way that once the coordinate is known, the particle is no longer in the state $\left|\mathbf{p}_{0}\right\rangle$ and the precise knowledge of its momentum is lost. One thus has to conclude that "when the momentum of a particle is known, its coordinate has no physical reality", since it does not satisfy the reality criterion. This example may be generalised to any observables $\widehat{P}$ and $\widehat{Q}$ which do not commute, since the corresponding physical quantities $P$ and $Q$ are subject to Heisenberg inequalities according to which one cannot know $P$ and $Q$ simultaneously with arbitrary accuracy. According to EPR, two alternatives follow: either (1) "the quantum-mechanical description of reality given by the wave function is not complete" or (2) [the quantum description is complete, but] "when the operators corresponding to two physical quantities do not commute the two quantities cannot have simultaneous reality".

\subsection{Incompleteness of quantum mechanics}

EPR consider that the second alternative leads to an inconsistency. For this purpose, assume a system of two particles 1 and 2 initially prepared without interaction, so that the wave function of the system is the product of two wave functions, the first one depending on the physical properties associated with particle 1 , the other depending on the physical properties of particle 2. Suppose now that the two particles start to interact during a finite time. According to Schrödinger equation, the wave function of the system is known at any time during and after the interaction. But usually, due to the interaction term, a mixing occurs and the wave function no longer appears as a product having the properties of the original one. The announced inconsistency arises from this entanglement of the particles state. A simple illustration of such an entangled state has been given by David Bohm [3], using two-state physical quantities. Consider a molecule made of two spin 1/2 atoms. The molecule is supposed to be prepared in a singlet state of total spin, $s=0$, represented by the entangled state

$$
|\psi\rangle=\frac{1}{\sqrt{2}}\left(\left|\uparrow_{z}\right\rangle \otimes\left|\downarrow_{z}\right\rangle-\left|\downarrow_{z}\right\rangle \otimes\left|\uparrow_{z}\right\rangle\right)
$$

where $\left|\uparrow_{z}\right\rangle \otimes\left|\downarrow_{z}\right\rangle$ is eigenstate of the observable $\widehat{S}_{1 z}$ with eigenvalue $+\hbar / 2$ and of observable $\widehat{S}_{2 z}$ with eigenvalue $-\hbar / 2^{1}$. At a given time, the molecule breaks up according to a process which does not change the initial total spin.

An experimentalist measures the spin component $S_{1 z}$ of atom 1. Suppose she or he gets the eigenvalue $+\hbar / 2$. According to the usual rules of quantum mechanics, the state vector $|\phi\rangle$ immediately after the measurement is the normalized projection of $|\psi\rangle$ on the eigenstate $|\uparrow z\rangle$ of $\widehat{S}_{1 z}$,

$$
|\phi\rangle=\left|\uparrow_{z}\right\rangle \otimes\left|\downarrow_{z}\right\rangle \text {. }
$$

\footnotetext{
${ }^{1}$ We use the conventional notation in which the left part of the tensor product refers to the first atom while the right part refers to the second atom.
} 
As a result of the measurement on atom 1, the state vector is disentangled and becomes a tensor product of individual state vectors associated with each atom. By the way, the state being proportional to $\cdots \otimes\left|\downarrow_{z}\right\rangle$, the spin component $S_{2 z}$ of atom 2 takes the value $-\hbar / 2$ with probability 1 . If the experimentalist had measured the spin component $S_{1 x}$ of atom 1 , and had obtained the value $+\hbar / 2$, then, immediately after measurement, the state vector would have become the normalized projection of $|\psi\rangle$ on $\left|\uparrow_{x}\right\rangle \otimes \ldots$,

$$
|\phi\rangle=\frac{1}{\sqrt{2}}\left|\uparrow_{x}\right\rangle \otimes\left\{\left|\downarrow_{z}\right\rangle-\left|\uparrow_{z}\right\rangle\right\}=-\left|\uparrow_{x}\right\rangle \otimes\left|\downarrow_{x}\right\rangle .
$$

It follows that a further measurement of the spin component $S_{2 x}$ of atom 2 leads to $-\hbar / 2$ with certainty $^{2}$.

We must conclude that in both cases, as far as no interaction has perturbed atom 2 since the disintegration, the state vectors $\cdots \otimes\left|\downarrow_{z}\right\rangle$ and $\cdots \otimes\left|\downarrow_{x}\right\rangle$ must be associated with a unique element of reality of this atom. As a consequence, according to the reality criterion of EPR, both spin components $S_{2 z}$ and $S_{2 x}$ should be simultaneously associated with an element of reality concerning atom 2 . Since it is well known that the observables $\widehat{S}_{2 z}$ and $\widehat{S}_{2 x}$ do not commute $\left(\left[\widehat{S}_{2 z}, \widehat{S}_{2 x}\right]=\mathrm{i} \hbar \widehat{S}_{2 y}\right)$, there is an inconsistency and one should come back to the first alternative: "the quantum-mechanical description of reality given by the wave function is not complete".

In their original paper, EPR pointed out that one would not reach the same conclusions if, in the reality criterion, one had required physical properties to be regarded as simultaneous elements of reality "only when they can be simultaneously measured or predicted". In this case, as shown in the previous example, the reality of the physical properties depends on the experiment achieved on atom 1, but as EPR concluded, "no reasonable definition of reality could be expected to permit this". This is nevertheless what Bohr would accept.

In their original paper, EPR discuss the example of two particles without interaction, prepared in a coordinate- and momentum- entangled state represented by the wave function

$$
\psi(1,2)=\int_{-\infty}^{+\infty} \exp \left[\mathrm{i}\left(x_{1}-x_{2}-X_{1}\right) p / \hbar\right] \mathrm{d} p
$$

where $X_{1}$ is a known constant. The contradiction lies in the fact that a precise measurement of the momentum of particle $1, p_{1}=p$, ensures that the momentum of particle 2 is known with certainty, $p_{2}=-p$, while a precise measurement of the coordinate of particle $1, x_{1}=x$, guarantees that particle 2 has coordinate $x_{2}=x-X_{1}$ with certainty. As far as particle 2 has not been perturbed since the preparation of the state, one concludes that its momentum and coordinate are known with certainty at a given time, in contradiction with Heisenberg inequalities.

\subsection{Bohr's reply}

In his paper of 1935 [4], Bohr reconsiders this example and elaborates upon the epistemology of complementarity, initiated during the 1927 Como and Bruxelles conference [5]. For that purpose, he points out that the EPR example is realized with a two-slit Young device. Consider two non-interacting particles 1 and 2 crossing simultaneously a two-slit apparatus, not rigidly attached to the support as represented in figure 1. One is then able to measure precisely the coordinate difference $X_{1}=x_{1}-x_{2}$ between the two particles at the time they cross it: this is the distance between the slits. Furthermore measuring the spring elongation or compression due to the crossing of the two particles, one is also able to deduce with arbitrary accuracy the total momentum transfered to the aperture along the $x$-axis, $P_{2}=p_{1}+p_{2}{ }^{3}$. One has thus prepared the EPR entangled state which corresponds to the particular case $P_{2}=0$.

\footnotetext{
${ }^{2}$ One should have obtained this result directly, since the spin of the system $1+2$ is zero. As a consequence, if the spin component of particle 1 is $+\hbar / 2$ along any direction, that of particle 2 along the same axis is $-\hbar / 2$.

${ }^{3}$ Indeed, $X_{1}$ and $P_{2}$ are not conjugate and might be measured at the same time with arbitrary accuracy. Considering pairs of conjugate variables $x_{1}, p_{1}$ and $x_{2}, p_{2}$ along $x$-axis, one may build other pairs of conjugate variables $X_{1}=x_{1}-x_{2}, P_{1}=p_{1}-p_{2}$ and $X_{2}=x_{1}+x_{2}, P_{2}=p_{1}+p_{2}$.
} 


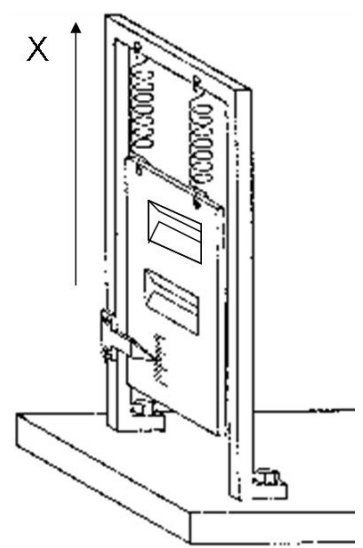

Figure 1. Double slit experimental setup.
A later precise measurement of the coordinate $\left(x_{1}\right)$ or of the momentum $\left(p_{1}\right)$ of particle 1 enables one to deduce the coordinate $\left(x_{2}\right)$ or momentum $\left(p_{2}\right)$ of particle 2 without perturbing it in any way. But, and this is where the reality criterion of EPR is unsuitable, one has to make a choice between the two experimental situations. This choice requires an unambiguous use of the classical concepts needed to determine the physical quantities. Accurate measuring of $x_{1}$, for instance, requires a well defined spatial frame in the classical sense, which needs the apparatus (a single slit aperture for example) to be rigidly fixed to the support that defines the reference frame. But in doing so, one drops out a further possibility to measure $p_{1}$, since the momentum transfer between the particle and the aperture is lost in the support. How then can an element of reality be associated with $p_{1}$, and thus with $p_{2}$, which are variables originating from classical concepts while we are unable to perform any measurements of them through the apparatus used, due to the very existence of the action quantum $h$. Measuring $p_{1}$, on the other hand, requires an experimental setup which us enables to use classical conservation laws of the momentum transfer between the particle 1 and the apparatus. This may be for instance the same single slit aperture, but now dissociated from the support, allowing for a measurement of the momentum through the elongation or compression of a spring. In doing so, we loose the ability of an accurate measurement of the coordinate $x_{1}$ (and thus $x_{2}$ ), the precise measurement of which requires a fixed frame of reference.

Therefore, according to Bohr, the reality criterion proposed by EPR contains an essential ambiguity when it is applied to atomic systems, ambiguity which lies in the words "without in any way disturbing a system". Of course in the example of EPR, there is no mechanical perturbation during the critical step of the measurement process, "but there is essentially the question of an influence on the very conditions which define the possible types of predictions regarding the future behaviour of the system." According to Bohr, the EPR argumentation is misleading, since it is based on the assumption that one may intelligibly speak about the state of a physical system, regardless of any experimental setup. This is equivalent to a discourse on the "the thing in itself", regardless of external conditions in which it is observed. According to Bohr, what quantum phenomena reveal, thanks to Heisenberg inequalities, is precisely the inability to define properties (elements of reality) of a quantum system without taking into account their interaction with other systems. He proposes a description of the quantum world in terms of complementarity, a way which although discussed in terms of classical concepts, accounts for the fact that no separation between the behaviour of atomic systems and their interaction with measurement devices is possible. This is complementarity. Wave and particle behaviours of light and matter are complementary in the sense that when a measurement is performed, one should use both classical concepts when interpretating in a consistent way the observed phenomena as a whole.

\subsection{Bohm's further explanations}

Although it plays an important role in the argumentation of EPR, Bohr did not extensively discuss the wave function. We shall follow Bohm to provide a complement to the orthodox interpretation of that point and of the significance of observables in quantum mechanics.

Firstly, one observes that the EPR argumentation is based on two implicit assumptions which are never discussed in the original paper. The first one is that the world may be correctly analyzed in terms of distinct elements of reality which have separate existence. This is the problem of separability, to which we shall return later. Secondly, elements of reality must have a definite mathematical correspondence in a complete theory. This second statement is in fact only a stronger version of the definition of elements of reality given by EPR, but it is this stronger version which is used in the paper, since the element of reality is defined even before the measurement. 
If one rejects both assumptions, which are at the basis of any classical theory, the paradox disappears. Indeed, orthodox quantum theory assumes that the strong correspondence stated above emerges at the classical level only. At a quantum level, mathematical description is provided through the wave function which is not in strong correspondence with the system, but in a statistical correspondence, although the wave function provides the most possible complete description of the system. In order to reconcile both seemingly contradictory aspects of the wave function, one has to accept that elements of reality of a quantum system do generally exist in an imprecise way. These are potentialities, to be likely realised when the interaction of the system with a classical measurement device occurs. The wave function describes the propagation of these correlated potentialities. Hence, concerning two non-commuting observables, such as the coordinate and the momentum of a particle, neither of them has a precise and definite existence. Rather, each of them is likely to be actualized when interactions with the adequate apparatus take place. Within this approach, the characteristics attributed to the observables are not intrinsic to the subsystem, but rather through their interaction in the complete system.

\subsection{Einstein's point of view on separability}

In his correspondence and in further papers published after the EPR article [6], Einstein clarified his point of view. The simple fact that the wave function describing particle 2 after measurement made on particle 1 depends on this measure is, according to him, the sign of incompleteness of quantum theory [7]:

"From the point of view of the [orthodox] interpretation this means that according to the choice of a complete measurement on $S_{1}$ a different real situation with respect to $S_{2}$ is created, which is described through differently natured $\psi_{2}, \psi_{2}^{\prime}, \psi_{2}^{\prime \prime}$ etc.

From the point of view of quantum mechanics alone this does not present any difficulty. According to the specific choice of the measurement on $S_{1}$ a distinct real situation is created and the necessity, to associate with the system $S_{2}$ at the same time two or more distinct wave functions $\psi_{2}, \psi_{2}^{\prime}, \ldots$, can never arise.

However, the situation changes if one tries to maintain, simultaneously with the principles of quantum mechanics, also the Principle of the independent existence of a real situation in two separated parts $R_{1}$ and $R_{2}$ of space. In our example a complete measurement on $S_{1}$ means a physical interaction which only concerns the part $R_{1}$ of space. Such an interaction, however, cannot directly influence the physical reality in a part $R_{2}$ of space which is far away. This would imply that any assertion with respect to $S_{2}$ which we could achieve through a complete measurement on $S_{1}$ must also be valid for the system $S_{2}$ when no measurement at all is made on $S_{1}$. That would mean that all statements on $S_{2}$ must be valid which can be derived by specifying $\psi_{2}$ or $\psi_{2}^{\prime}$ etc. This is of course impossible, if $\psi_{2}, \psi_{2}^{\prime}$ etc. stand for mutually distinct real facts of $S_{2}$, that is one enters in conflict with the [orthodox] interpretation of the $\psi$-function.

Without doubt, it appears to me that the physicists that take the description of quantum mechanics for definitive in principle, will react to this thought as follows: they will drop the requirement of the independent existence of the Physical Reality present in different parts of space; they are justified in pointing out that Quantum Theory nowhere makes use of this requirement.

I admit this, but point out: if I consider the physical phenomena I know about, in particular those which are accounted for so successfully by quantum mechanics, I still find nowhere a fact which would make it probable to me that the requirement [of separability] should be abandoned. I am therefore inclined to believe that in the sense of [orthodox interpretation] the description of quantum mechanics should be viewed as an incomplete and indirect description of reality, which will be replaced later by a complete and direct one."

\subsection{What orthodox physicists should accept, and what others may reject}

In 1935 Schrödinger cat paradox also appeared. A cat enclosed in a box plays the role of a measurement apparatus with two macroscopic mutually exclusive outcomes, dead or alive. The answer is given in modern quantum mechanics through the concept of quantum decoherence of 
entangled macroscopic states. This notion is a bit out of the scope of the present contribution ${ }^{4}$, but is required to justify epistemological completeness of orthodox quantum theory.

As a summary, an orthodox physicist has to admit that physical properties attributed to a quantum system are only potentialities which become physical reality when interacting with a measurement apparatus. For such a physicist, the concept of trajectory of a particle is meaningless, unless a device is used to measure it. He (she) also admits that "nature plays dice", that is probabilities are inherent to quantum phenomena. Those who do not share this opinion can invoke some hidden variables at the origin of probabilities which would then reveal our ignorance of these variables. In this direction, one has to distinguish between non-local hidden-variable theories (Bohm - de Broglie theory is the paradigmatic example) and local hidden-variable theories which are discussed below in this paper. The question of separability is nevertheless still present.

\section{On the impossibility of local hidden variables}

\subsection{Hidden-variable theories}

In classical physics, measurement processes are intuitive: the result of the measurement of any physical observable $\mathcal{A}$ is a function of the state of the system, e.g. the positions $\mathbf{r}_{i}$ and the velocities $\mathbf{v}_{i}$ for a set a particles. The measurement of $\mathcal{A}$ gives the same result in all systems identically prepared. For this reason, the classical state is said to be a dispersion-free state. In contradistinction, quantum states are not dispersion-free. For systems identically prepared in a given quantum state $|\psi\rangle$, the measurement of $\mathcal{A}$ does not give a unique result but one of the eigenvalues $a_{i}$ of the associated hermitian operator $\widehat{A}$ with the probability $\left|\left\langle\psi \mid \phi_{i}\right\rangle\right|^{2}$ if $\widehat{A}\left|\phi_{i}\right\rangle=a_{i}\left|\phi_{i}\right\rangle$. When the number of such systems becomes large, the average measurement is thus $\langle A\rangle=\langle\psi|\widehat{A}| \psi\rangle$ and the mean dispersion around this average is $\sigma_{A}=\sqrt{\left\langle\psi\left|\widehat{A}^{2}\right| \psi\right\rangle-\langle\psi|\widehat{A}| \psi\rangle^{2}}$. The quantum state $|\psi\rangle$ is not sufficient to determine the output of a unique measurement of $\mathcal{A}$. According to the orthodox interpretation of quantum mechanics, this uncertainty on the output of an unique measurement is a fundamental property of quantum world.

It is tempting, as Einstein, Podolsky and Rosen did, to make the assumption that the wave function $|\psi\rangle$ does not give a complete description of the state of the system. There may exist a set of hidden variables $\lambda$, not yet experimentally observed, such that $\{|\psi\rangle, \lambda\}$ completely determines the output of a unique measurement, i.e. that $\{|\psi\rangle, \lambda\}$ is a dispersion-free state. Let $V_{\{|\psi\rangle, \lambda\}}(\widehat{A})$ be the output of a unique measurement on a system in the complete state $\{|\psi\rangle, \lambda\}$. The function $V$ maps the dispersion-free states $\{|\psi\rangle, \lambda\}$ onto the eigenvalues of the operator $\widehat{A}$. Since the variables $\lambda$ are unknown, one cannot prepare experimentally different systems in the same state $\{|\psi\rangle, \lambda\}$. One can only ensure that the wave function $|\psi\rangle$ is the same, but the hidden variables $\lambda$ remain uncontrolled for whatever reason ${ }^{5}$ in an ensemble of different systems. Let $\wp(\lambda)$ be the probability distribution of the hidden variables among the different systems. When the number of such systems becomes large, the average measurement of $\mathcal{A}$ should be equal to the prediction of the quantum theory:

$$
\langle A\rangle=\int V_{\{|\psi\rangle, \lambda\}}(\widehat{A}) \wp(\lambda) \mathrm{d} \lambda=\langle\psi|\widehat{A}| \psi\rangle
$$

Another important difference to quantum mechanics is that the wave packet reduction postulate is not necessary anymore. In order to explain the empirical fact that the same output $a_{i}$ is obtained when repeating the same measurement $\mathcal{A}$ on the same system, one needs to assume in quantum mechanics that the wave function $|\psi\rangle$ of the system is projected onto the eigenvector $\left|\phi_{i}\right\rangle$ of $\widehat{A}$ associated with the eigenvalue $a_{i}$. In hidden-variable theories, it is sufficient to assume that the

\footnotetext{
${ }^{4}$ Quantum decoherence is the phenomenon according to which the time evolution of a quantum superposition of states, when interacting with an environment, displays interference terms which are amazingly rapidly suppressed at a time scale which is by orders of magnitude shorter than the typical relaxation times of the system.

${ }^{5}$ They may depend on the history of the system, non-separability with the rest of the Universe, intrinsic stochasticity, etc.
} 
complete state $\{|\psi\rangle, \lambda\}$ is not modified during the measurement process. That is the usual classical measurement picture.

\section{2. von Neumann no-go theorems}

In the very early years of quantum theory, von Neumann studied the possibility of an underlying hidden-variable quantum theory [8]. He showed that the linearity postulate of quantum mechanics, implying

$$
\langle(\alpha A+\beta B)\rangle=\alpha\langle A\rangle+\beta\langle B\rangle,
$$

cannot be fulfilled by a hidden-variable theory, which demonstrates according to him, the impossibility of such a theory. In the following, we give an outline of his demonstration (for further details, see for instance the excellent dissertation by D. Hemmick [9] on which this section is based). First, von Neumann showed that equation (6) implies that $\langle A\rangle=\operatorname{Tr} \widehat{A} \hat{\rho}$ where $\hat{\rho}$ is a density operator equal to $\hat{\rho}=|\psi\rangle\langle\psi|$ in quantum mechanics. Invoking equation (5), one can show that (6) also applies to $V_{\{|\psi\rangle, \lambda\}}(\widehat{A})$, i.e.

$$
V_{\{|\psi\rangle, \lambda\}}(\alpha A+\beta B)=\alpha V_{\{|\psi\rangle, \lambda\}}(A)+\beta V_{\{|\psi\rangle, \lambda\}}(B),
$$

and thus $V_{\{|\psi\rangle, \lambda\}}(A)=\operatorname{Tr} \hat{\rho}_{\lambda} \widehat{A}$. Applying this relation to the projector $\widehat{P}_{\phi}=|\phi\rangle\langle\phi|$, one obtains

$$
V_{\{|\psi\rangle, \lambda\}}\left(\widehat{P}_{\phi}\right)=\operatorname{Tr} \hat{\rho}_{\lambda}|\phi\rangle\langle\phi|=\left\langle\phi\left|\rho_{\lambda}\right| \phi\right\rangle .
$$

The measurement output $V_{\{|\psi\rangle, \lambda\}}\left(\widehat{P}_{\phi}\right)$ is simply a matrix element of $\hat{\rho}_{\lambda}$. On the other hand, since $\widehat{P}_{\phi}^{2}=\widehat{P}_{\phi}$, and since repeating twice the measurement should give twice the same output in a classical measurement process, one has the relation

$$
V_{\{|\psi\rangle, \lambda\}}\left(\widehat{P}_{\phi}\right)=V_{\{|\psi\rangle, \lambda\}}\left(\widehat{P}_{\phi}^{2}\right)=\left[V_{\{|\psi\rangle, \lambda\}}\left(\widehat{P}_{\phi}\right)\right]^{2},
$$

which implies that $V_{\{|\psi\rangle, \lambda\}}\left(\widehat{P}_{\phi}\right)$ is equal to 0 or 1 . The vector $|\phi\rangle$ being arbitrary, we are led to the conclusion that all matrix elements of $\hat{\rho}_{\lambda}$ are either 0 or 1 , in contradiction with $\operatorname{Tr} \rho_{\lambda}=1$.

The correctness of the von Neumann demonstration is not questionable but one may wonder whether the postulate (7) is really necessary. The alternative demonstration of the von Neumann theorem provided by Schrödinger is helpful to address this question [10]. Consider a particle in a harmonic potential with Hamiltonian

$$
\widehat{H}=\frac{\hat{p}^{2}}{2 m}+\frac{1}{2} m \omega^{2} \hat{x}^{2}
$$

Equation (7) reads for $\widehat{H}$,

$$
\begin{aligned}
V_{\{|\psi\rangle, \lambda\}}(\widehat{H}) & =\frac{1}{2 m} V_{\{|\psi\rangle, \lambda\}}\left(\hat{p}^{2}\right)+\frac{1}{2} m \omega^{2} V_{\{|\psi\rangle, \lambda\}}\left(\hat{x}^{2}\right) \\
& =\frac{1}{2 m}\left[V_{\{|\psi\rangle, \lambda\}}(\hat{p})\right]^{2}+\frac{1}{2} m \omega^{2}\left[V_{\{|\psi\rangle, \lambda\}}(\hat{x})\right]^{2}
\end{aligned}
$$

Since in a given experiment $V_{\{|\psi\rangle, \lambda\}}(\hat{x})$ and $V_{\{|\psi\rangle, \lambda\}}(\hat{p})$ can take any real value, it is not possible to ensure that $V_{\{|\psi\rangle, \lambda\}}(\widehat{H})$ is always equal to one of the discrete eigenvalues $\hbar \omega(n+1 / 2)$ of $\widehat{H}$. Unlike von Neumann, Schrödinger questioned the validity of the postulate (7). Indeed, $\hat{x}$ and $\hat{p}$ are non-commuting operators, i.e. $[\hat{x}, \hat{p}]=\mathrm{i} \hbar$, which means, according to Heisenberg uncertainty principle, that position and velocity cannot be measured during the same experiment with arbitrary accuracy. As a consequence, $V_{\{|\psi\rangle, \lambda\}}(\hat{x})$ and $V_{\{|\psi\rangle, \lambda\}}(\hat{p})$ have no simultaneous meaning. Two different experiments have to be performed to measure both position and velocity. The postulate (7) is not applicable to non-commuting operators but only to sets of commuting ones, i.e. to quantities that can be measured during the same experiment. Using the same approach as von Neumann, Gleason showed that restricting the postulate (7) only to sets of commuting operators leads to 
the same conclusion: the impossibility of such hidden-variable theory [11]. Kochen and Specker obtained the same result in the case of a spin one particle [12]. However, as the latter recognised by Bell, one can find operators such that $[\widehat{A}, \widehat{B}]=0$ and $[\widehat{A}, \widehat{C}]=0$ but $[\widehat{B}, \widehat{C}] \neq 0$ which means that both $\mathcal{A}$ and $\mathcal{B}$ can be measured with arbitrary accuracy with a given experimental setup, $\mathcal{A}$ and $\mathcal{C}$ can be measured similarly with a second experimental setup but no experimental setup allows the simultaneous precise measurement of $\mathcal{B}$ and $\mathcal{C}$. As a consequence, the mapping function $V_{\{|\psi\rangle, \lambda\}}(\widehat{A})$ giving the output of a unique measurement of $\mathcal{A}$ should depend on the experimental setup used. This obvious property is called contextuality.

\subsection{Bell inequalities}

Consider once more Bohm's version of EPR experiment. The system is described in quantum mechanics by the singlet state (1). The spins are sent away from each other and are measured in two different directions $\mathbf{a}$ and $\mathbf{b}$ at the same time, or at least at times sufficiently close to forbid any communication (at the speed of light) between the two measurements. The outputs $\sigma_{1}^{\mathbf{a}}=\boldsymbol{\sigma}_{1}$.a and $\sigma_{2}^{\mathbf{b}}=\boldsymbol{\sigma}_{b} . \mathbf{b}$ of the measurements are multiplied and averaged over a large number of experiments, leading to a correlation function $C(\mathbf{a}, \mathbf{b})=\left\langle\sigma_{1}^{\mathbf{a}} \sigma_{2}^{\mathbf{b}}\right\rangle$. Quantum mechanics predicts that this correlation function is equal to

$$
C_{Q M}(\mathbf{a}, \mathbf{b})=-\mathbf{a} \cdot \mathbf{b} .
$$

Note that if the two spins are measured in the same direction $\mathbf{a}=\mathbf{b}$, the correlation is simply $C_{Q M}(\mathbf{a}, \mathbf{a})=-1$ which means that in all experiments, the two spins have always been observed in opposite directions, reflecting the fact that the total spin of the system is zero.

One is tempted to think in terms of classical vectors, but Bell showed that this picture is incompatible with the average correlation (12) predicted by quantum mechanics [13]. Indeed, in a local hidden-variable theory, the average correlation reads

$$
C(\mathbf{a}, \mathbf{b})=\int \mathrm{d} \lambda \wp(\lambda) A(\lambda, \mathbf{a}) B(\lambda, \mathbf{b}),
$$

where $A(\lambda, \mathbf{a})=V_{\{|\psi\rangle, \lambda\}}\left(\sigma_{1}^{\mathbf{a}}\right)= \pm 1$ and $B(\lambda, \mathbf{b})=V_{\{|\psi\rangle, \lambda\}}\left(\sigma_{2}^{\mathbf{b}}\right)= \pm 1$. Note that this expression satisfies the requirement of locality: the measurement of $\sigma_{1}^{\mathbf{a}}$ for instance does not affect that of $\sigma_{1}^{\mathbf{b}} 6$. Since the total spin is zero and $C(\mathbf{a}, \mathbf{a})=-1$, one can write

$$
-1=\int \mathrm{d} \lambda \wp(\lambda) A(\lambda, \mathbf{a}) B(\lambda, \mathbf{a}) \Leftrightarrow A(\lambda, \mathbf{a})=-B^{-1}(\lambda, \mathbf{a})=-B(\lambda, \mathbf{a}) .
$$

As a consequence, the difference of the average correlations measured in the directions of vectors $\mathbf{a}$ and $\mathbf{b}$ and then $\mathbf{a}$ and $\mathbf{c}$ is

$$
\begin{aligned}
C(\mathbf{a}, \mathbf{b})-C(\mathbf{a}, \mathbf{c}) & =-\int \mathrm{d} \lambda \wp(\lambda)[A(\lambda, \mathbf{a}) A(\lambda, \mathbf{b})-A(\lambda, \mathbf{a}) A(\lambda, \mathbf{c})] \\
& =-\int \mathrm{d} \lambda \wp(\lambda) A(\lambda, \mathbf{a}) A(\lambda, \mathbf{b})\left[1-A^{-1}(\lambda, \mathbf{b}) A(\lambda, \mathbf{c})\right] \\
& =-\int \mathrm{d} \lambda \wp(\lambda) A(\lambda, \mathbf{a}) A(\lambda, \mathbf{b})[1-A(\lambda, \mathbf{b}) A(\lambda, \mathbf{c})],
\end{aligned}
$$

and, since $A(\lambda, \mathbf{a})= \pm 1$

$$
\begin{aligned}
|C(\mathbf{a}, \mathbf{b})-C(\mathbf{a}, \mathbf{c})| & \leqslant \int \mathrm{d} \lambda \wp(\lambda)|A(\lambda, \mathbf{a}) A(\lambda, \mathbf{b})|[1-A(\lambda, \mathbf{b}) A(\lambda, \mathbf{c})] \\
& \leqslant \int \mathrm{d} \lambda \wp(\lambda)[1-A(\lambda, \mathbf{b}) A(\lambda, \mathbf{c})] \\
& \leqslant \int \mathrm{d} \lambda \wp(\lambda)[1+A(\lambda, \mathbf{b}) B(\lambda, \mathbf{c})] .
\end{aligned}
$$

\footnotetext{
${ }^{6}$ In the case of simultaneous measurements, a non-local hidden-theory correlation would be written as $C(\mathbf{a}, \mathbf{b})=$ $\int \mathrm{d} \lambda \wp(\lambda) A(\lambda, \mathbf{a}, \mathbf{b}) B(\lambda, \mathbf{a}, \mathbf{b})$
} 
Any local hidden-variable theory should then satisfy the so-called Bell inequalities:

$$
|C(\mathbf{a}, \mathbf{b})-C(\mathbf{a}, \mathbf{c})| \leqslant 1+C(\mathbf{b}, \mathbf{c})
$$

for any set of vectors $\mathbf{a}, \mathbf{b}$ and $\mathbf{c}$. In contradistinction, these inequalities are violated by quantum mechanics as one can check by inserting the quantum correlation (12) into (17). The experimental test of the Bell inequalities (see next section) allows us to decide unambiguously in favour of either quantum mechanics or local hidden-variable classical theory. Note that we have restricted our attention to local theories thus non-local hidden-variable theories may violate the Bell inequalities too.

\subsection{A simple illustration of Bell's inequalities}

As a simple illustration, consider the following local hidden-variable theory. Spins are unit vectors and the measurement of $\boldsymbol{\sigma}$.a gives in a deterministic way the sign of $\boldsymbol{\sigma}$.a. Equivalently, the state of a spin can be associated with a point on the unit sphere and the measurement of $\boldsymbol{\sigma}$.a gives +1 if this point is in the hemisphere whose revolution axes is along a and -1 otherwise (see leftmost illustration of the figure 2). An entangled pair is supposed to be made of two spins in opposite directions so that $\boldsymbol{\sigma}_{2}=-\boldsymbol{\sigma}_{1}$. The measurement of $\left(\boldsymbol{\sigma}_{1} \cdot \mathbf{a}\right)\left(\boldsymbol{\sigma}_{2} \cdot \mathbf{b}\right)=-\left(\boldsymbol{\sigma}_{1} \cdot \mathbf{a}\right)\left(\boldsymbol{\sigma}_{1} \cdot \mathbf{b}\right)$ gives +1 if $\boldsymbol{\sigma}_{1}$ is only in one of the two hemispheres whose revolutions axes are respectively along a and $\mathbf{b}$, and -1 otherwise. As can be seen in the central diagram of figure 2 , the total surface giving an output +1 is $4 \theta$ where $\theta$ is the angle between the two vectors $\mathbf{a}$ and $\mathbf{b}$. When assuming that the spins are produced in any direction $\boldsymbol{\sigma}_{1}=-\boldsymbol{\sigma}_{2}$, the average correlation is

$$
C(\mathbf{a}, \mathbf{b})=\frac{1}{4 \pi}[+1 \times 4 \theta+(-1) \times 4(\pi-\theta)]=\frac{2 \theta}{\pi}-1 .
$$

The Bell inequalities are satisfied:

$$
|C(\mathbf{a}, \mathbf{b})-C(\mathbf{a}, \mathbf{c})|=\frac{2}{\pi}|\theta-\varphi| \leqslant \frac{2 \phi}{\pi},
$$

where $\varphi$ is the angle between $\mathbf{a}$ and $\mathbf{c}$ and $\phi$ between $\mathbf{b}$ and $\mathbf{c}$. Note that the angles $\theta, \varphi$ and $\phi$ are the length of the three edges of the triangle on the unit sphere joining the vectors $\mathbf{a}, \mathbf{b}$ and $\mathbf{c}$ (see the rightmost illustration of figure 2). The equality is obtained when the three vectors are in the same plane.
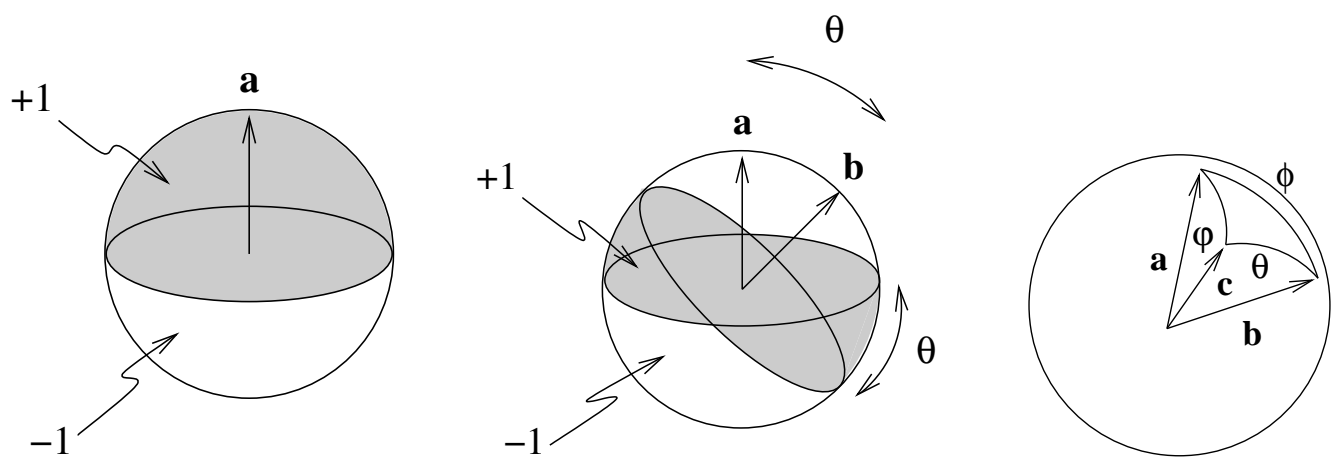

Figure 2. Illustration of the measurement in the hidden-variable theory considered in the text.

\section{Tests of Bell's inequalities: from thought experiments to realistic experi- ments}

The conclusion of the above paragraph is that the Bell inequalities provide a quantitative criterion to test the hidden-variable theories versus quantum mechanics. Bell's inequalities brought 


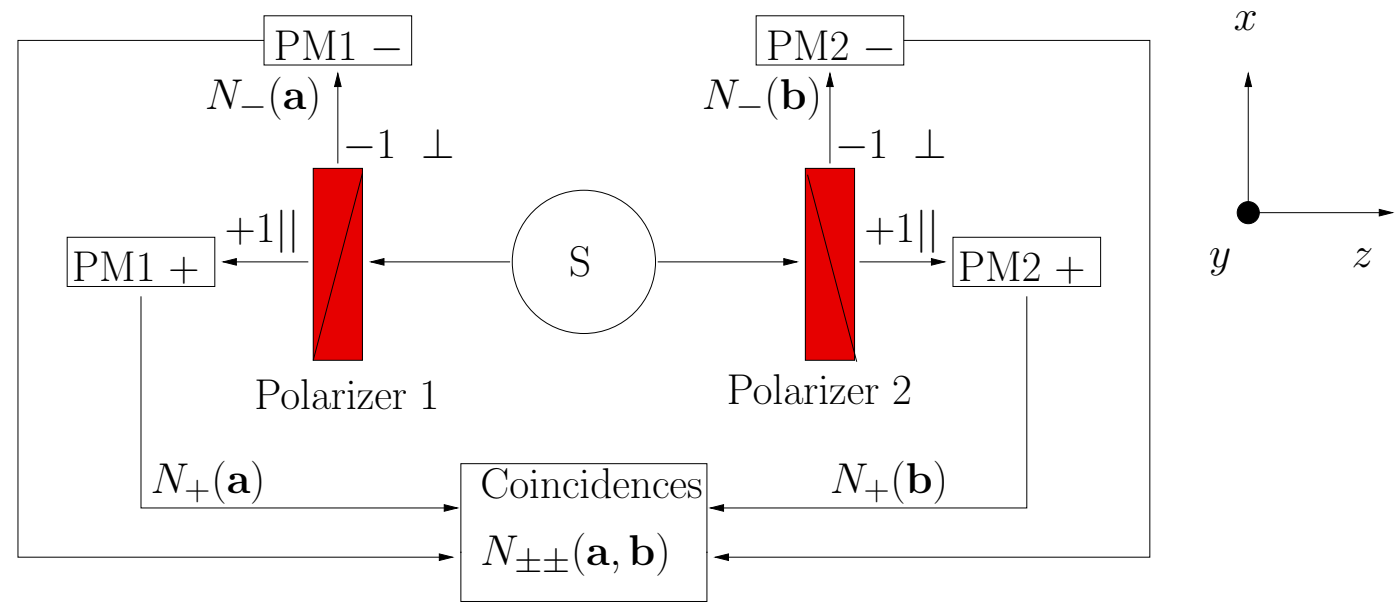

Figure 3. Optical version of the Bohm experiment. $\mathrm{S}$ is the source of photons. PM are photomultipliers.

the discussion into the experimental domain. Many experiments have since been performed, the outcomes of which are consistent with quantum mechanics and inconsistent with local realism.

The first step through experimental realization was performed in 1969 by Clauser, Horne, Shimony and Holt [14]. They generalized the Bell theorem so as to apply to actual experiments (CHSH inequalities). At the end of their paper, they proposed an experiment based on the Bohm and Aharonov [15] version of the EPR paradox. It is an optical variant aimed at measuring the polarization correlation of a pair of optical photons. This experiment was the first of a long series in quantum optics.

The two main assumptions underlying the formalism leading to Bell's theorem are:

i) Existence of hidden variables which renders an account of the correlations. It is a necessary hypothesis to obtain a conflict between the Bell inequalities and quantum mechanics.

ii) Locality: the result of a measurement of the polarization of one of the photons does not depend on the orientation of the other analyzer and vice-versa and the way the pairs are emitted by the source does not depend on the measurement setup. The locality assumption is thus crucial: Bell's inequalities will no longer hold without it.

Let us stress that determinism does not seem to be a necessary ingredient, since it is possible to obtain analogous inequalities with local stochastic hidden-variable theories [16,17]. Indeed, in 1969 Bell himself gave an example where the local hidden variables do not completely determine the outcome of the measurements. One can imagine for example that, for whatever reason, some local fluctuations occur and are responsible for the stochastic character of the measurement outcomes. However such a theory is still in conflict with quantum mechanics.

\subsection{Optical variant of the Bohm experiment}

Figure 3 illustrates the optical variant [18] of the Bohm version [15] of the EPR thought experiment. The source $S$ produces pairs of photons with different frequencies $\nu_{1}$ and $\nu_{2}$, sent in opposite directions. Each photon encounters an analyzer (a polarizer able to measure the linear polarization) whose orientation can be set by the experimentalist. The emerging signals are detected by photo-multipliers along two perpendicular directions. A coincidence monitor measures 
the probabilities of single or joint detections. The analyzer I (respectively analyzer II) is oriented along the $\mathbf{a}$ (respectively $\mathbf{b}$ ) direction. The result of the measurement of photon $\nu_{1}$ is $A(\mathbf{a})=+1$ if the polarization is parallel to $\mathbf{a}$ and -1 if the polarization is perpendicular to $\mathbf{a}$. The result of the measurement of photon $\nu_{2}$ is $B(\mathbf{b})=+1$ if the polarization is parallel to $\mathbf{b}$ and -1 if the polarization is perpendicular to $\mathbf{b}$.

The polarization part of the state vector describing the pair is an entangled state:

$$
|\psi(1,2)\rangle=\frac{1}{\sqrt{2}}[|x, x\rangle+|y, y\rangle]
$$

where $|x\rangle$ and $|y\rangle$ are one-photon linear polarizations states. The polarization correlation coefficient for polarizers in orientations $\mathbf{a}$ and $\mathbf{b}$ is

$$
\begin{aligned}
C(\mathbf{a}, \mathbf{b}) & =\left[N_{++}(\mathbf{a}, \mathbf{b})+N_{--}(\mathbf{a}, \mathbf{b})-N_{-+}(\mathbf{a}, \mathbf{b})-N_{+-}(\mathbf{a}, \mathbf{b})\right] / N \\
& =P_{++}(\mathbf{a}, \mathbf{b})+P_{--}(\mathbf{a}, \mathbf{b})-P_{-+}(\mathbf{a}, \mathbf{b})-P_{+-}(\mathbf{a}, \mathbf{b})
\end{aligned}
$$

where $N$ is the rate of emission of pairs, $N_{ \pm \pm}(\mathbf{a}, \mathbf{b})$ are the coincidence rates and $P_{ \pm \pm}(\mathbf{a}, \mathbf{b})$ the empirical probabilities of joint detection. Using the entangled state (20), the Bell inequality reads as

$$
|C(\mathbf{a}, \mathbf{b})-C(\mathbf{a}, \mathbf{c})| \leqslant 1-C(\mathbf{b}, \mathbf{c}) .
$$

\subsection{Towards experimental tests: the $\mathrm{CHSH}$ inequalities}

In 1969, Clauser, Horne, Shimony and Holt [14] expressed the Bell inequalities in terms of experimental quantities, namely coincidence rates measured for 4 directions $\mathbf{a}, \mathbf{a}^{\prime}, \mathbf{b}$, and $\mathbf{b}^{\prime}$ of the polarizers,

$$
-2 \leqslant S \leqslant 2 \quad \text { with } \quad S=\left|C(\mathbf{a}, \mathbf{b})-C\left(\mathbf{a}, \mathbf{b}^{\prime}\right)\right|+C\left(\mathbf{a}^{\prime}, \mathbf{b}\right)+C\left(\mathbf{a}^{\prime}, \mathbf{b}^{\prime}\right)
$$

Using quantum mechanics, the probabilities of detections are

$$
\begin{aligned}
P_{+}(\mathbf{a}) & =P_{+}(\mathbf{b})=P_{-}(\mathbf{a})=P_{-}(\mathbf{b})=\frac{1}{2} \\
P_{++}(\mathbf{a}, \mathbf{b}) & =P_{--}(\mathbf{a}, \mathbf{b})=\frac{1}{2} \cos ^{2}(\mathbf{a}, \mathbf{b}) \\
P_{+-}(\mathbf{a}, \mathbf{b}) & =P_{-+}(\mathbf{a}, \mathbf{b})=\frac{1}{2} \sin ^{2}(\mathbf{a}, \mathbf{b})
\end{aligned}
$$

leading to the correlation coefficient

$$
C_{Q M}(\mathbf{a}, \mathbf{b})=\cos 2(\mathbf{a}, \mathbf{b})
$$

The quantum mechanical expression for $S$ is thus

$$
S_{Q M}(\mathbf{a}, \mathbf{b})=\cos 2 \theta+\cos 2 \theta^{\prime}+\cos 2 \theta^{\prime \prime}-\cos 2\left(\theta+\theta^{\prime}+\theta^{\prime \prime}\right)
$$

with $(\mathbf{a}, \mathbf{b})=\theta,\left(\mathbf{b}, \mathbf{a}^{\prime}\right)=\theta^{\prime},\left(\mathbf{a}^{\prime}, \mathbf{b}^{\prime}\right)=\theta^{\prime \prime},\left(\mathbf{a}, \mathbf{b}^{\prime}\right)=\theta+\theta^{\prime}+\theta^{\prime \prime}$. The variation of $S_{Q M}$ when $\theta=\theta^{\prime}=\theta^{\prime \prime}$ is shown in figure 4. The conflicts with CHSH (23) inequalities occur when the absolute value of $|S|$ is larger than $2\left(S_{Q M}^{\max }=2 \sqrt{2}\right.$ and $\left.S_{Q M}^{\min }=-2 \sqrt{2}\right)$. However, it is clear that there are many orientations for which there is no conflict.

However, in the 70's, two-channel polarizers were not available and experiments were performed with one channel polarizers. Such polarizers transmit light polarized parallel to a (or $\mathbf{b}$ ), but not the orthogonal counterpart so that only the $N_{+}(\mathbf{a}), N_{+}(\mathbf{b})$ counting rates and the $N_{++}(\mathbf{a}, \mathbf{b})$ coincidence rate are measured. The above Bell inequalities have to be formulated again in order to depend only on these measured quantities. Moreover, in realistic experiments only $10^{-5}$ of the emitted photons are detected, so the quantities involved in $S$ are not of the same order of magnitude and there is no more possibility of violation of the generalized Bell inequalities. In fact, one needs 


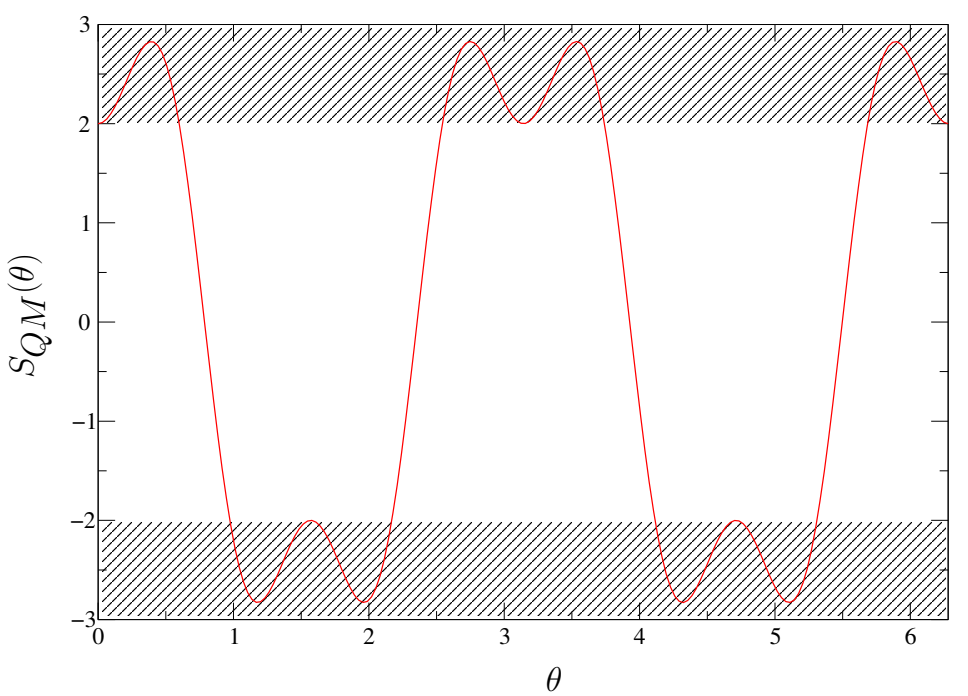

Figure 4. $\mathrm{S}_{Q M}$ versus $\theta$ as predicted by quantum mechanics for EPR pairs. The conflict with Bell inequalities happens when $|\mathrm{S}|$ is larger than 2.

to consider only the pairs actually detected. To do so, it is necessary to measure the rates when polarizers are removed. The final version of the CHSH inequality is then

$$
0 \leqslant S^{\prime} \leqslant 1,
$$

with

$$
\begin{aligned}
S^{\prime}= & \frac{1}{N(\infty, \infty)}\left[N_{++}(\mathbf{a}, \mathbf{b})-N_{++}\left(\mathbf{a}, \mathbf{b}^{\prime}\right)+N_{++}\left(\mathbf{a}^{\prime}, \mathbf{b}\right)\right. \\
& \left.+N_{++}\left(\mathbf{a}^{\prime}, \mathbf{b}^{\prime}\right)-N_{++}\left(\mathbf{a}^{\prime}, \infty\right)-N_{++}(\infty, \mathbf{b})\right] .
\end{aligned}
$$

The symbol $\infty$ conventionally indicates the absence of a polarizer. The important point is that $S^{\prime}$ now depends only on measured quantities of the same order of magnitude. For single channel polarizers, $S_{Q M}^{\prime} \max =0.207$ and $S_{Q M}^{\prime \min }=-1.207$ correspond to the maximum conflict.

Experimental inefficiencies (polarizer defects including non-perfect transmission rates, nonfinite detection angle, accidental birefringence, etc.) lead to a decrease of $C(\mathbf{a}, \mathbf{b}) . S_{Q M}\left(\mathbf{a}, \mathbf{a}^{\prime}, \mathbf{b}, \mathbf{b}^{\prime}\right)$ or $S_{Q M}^{\prime}\left(\mathbf{a}, \mathbf{a}^{\prime}, \mathbf{b}, \mathbf{b}^{\prime}\right)$ is then reduced, hence eventually the conflict with Bell's inequalities may be undetectable. Actual transmission coefficients and finite solid angles lead to $S_{Q M}^{\prime} \max =0.06$ and $S_{Q M}^{\prime} \min =-1.06$. A sensitive test with one channel polarizers is thus possible if the actual experiment is as close as possible to the thought experiment.

\subsection{First experiments in the 70 's}

Following the CHSH paper, a first series of tests was performed in the 70's using polarizationcorrelated visible photons. The entangled pair is produced by atomic radiative cascades. A beam of atoms is emitted by an oven in a vacuum chamber and submitted to an excitation. During the de-excitation process, two photons are produced nearly simultaneously if the lifetime of the intermediate level is short enough. For example, for the 0-1-0 calcium cascade $\left(4 p^{2}{ }^{1} S_{0} \rightarrow 4 s 4 p\right.$ $\left.{ }^{1} P_{1} \rightarrow 4 s^{2}{ }^{1} S_{0}\right)$ a green and a violet photon are produced and the lifetime of the intermediate level is very short, namely $5 \mathrm{~ns}$.

A first experiment was performed by Clauser and Freedman in Berkeley in 1972 [19]. They use the 0-1-0 Ca cascade described above. The excited state was obtained by ultraviolet radiation. In fact, additional spurious cascades occur and, because of consequently weak signal, the experiment took 200 hours. A violation of Bell's inequalities by 5 standard deviations was observed. 
Experiments have also been performed using a 1-1-0 cascade of mercury. The first one was in disagreement with quantum mechanics [20]. However, the experiment reproduced by another group leads to a significant violation of Bell's inequalities [21]. This last experiment took 412 hours. Finally, in 1976, Fry and Thompson [22] produce entangled pairs with a rate of several orders of magnitude larger. To obtain such a gain, they excited the upper level selectively using a laser. The data were collected in 80 minutes and were in excellent agreement with quantum mechanics, within 4 standard deviations.

Thus, at the end of the 70's, the experiments were already in agreement with quantum mechanics. However, they were performed with single channel polarizers and one has to keep in mind that their use requires indirect reasoning, and auxiliary calibrations.

\subsection{Experiments of the Orsay group (1981-1982)}

Thanks to progress in laser physics and modern optics, a second generation of experiments was carried out by the Orsay group in the early 80's. They developed a high efficiency stable and well controlled source of entangled photons. Using the same calcium cascade as Freedman et al, Aspect et al performed direct excitation using two lasers, so that the atom radiative decay delivered only pairs of entangled photons.

The first experiment was performed using single channel polarizers [23], with an improved analyzer $^{7}$ ensuring excellent rotational invariance and good transmission coefficients. Moreover, the main difference with previous experiments was that the excitation rate was more than ten times larger, allowing a large variety of tests to be performed with a good statistical accuracy. Firstly they performed measurements for the full $360^{\circ}$ range of relative orientation of the polarizers. The experimental value of $S^{\prime}$ was found equal to $0.126 \pm 0.014$ violating the Bell inequalities by 9 standard deviations and in excellent agreement with the value calculated from quantum mechanics including the polarizers efficiencies and the lense aperture angles, $0.118 \pm 0.005$. Secondly, no change was observed in the results with polarizers at a distance $(6 \mathrm{~m})$ larger than the coherence length of $\nu_{2}(1.5 \mathrm{~m})$.

The second experiment of the Orsay group, using two-channel polarizers ${ }^{8}$ [24] was the first actual analog of a Stern-Gerlach filter for spin 1/2 particles. Five runs were performed for each sensitive orientation of the polarizers. The average yielded $S=2.697 \pm 0.015$ in very good agreement with quantum mechanics $\left(S_{Q M}=2.70 \pm 0.05\right)$. This result leads to the greatest violation of generalized Bell inequalities ever achieved (40 standard deviations!).

All the above experiments were static experiments: the locality condition - which is crucial - is indirectly tested. In 1964, J.S Bell already noted that "the settings of the instruments are made sufficiently in advance to allow them to reach some mutual rapport by exchange of signals with velocity less than or equal to that of light". Consequently, the important point was to perform an experiment in which the settings are changed during the flight of the particles. The locality condition would then be compatible with Einstein causality preventing any faster-than-light effect. Such a timing experiment was done by the Orsay group in 1982 [25]. During this third experiment, the setup was modified in order to switch the direction of polarization analysis after the photons left the source. To do so, the (single-channel) polarizers were replaced by a switching device together with two polarizers in two different orientations, equivalent to a variable polarizer switched between two polarizations. The switchings are periodic, but the relative phases were randomly chosen and uncorrelated. The distance between the switches was large enough $(13 \mathrm{~m})$ for the time of travel of a signal at the velocity of light (43 ns) to be significantly larger than the delay between two switchings (about $10 \mathrm{~ns}$ ) and the delay between the emission of the two photons (5ns average).

Because of a reduced signal (due to the limited aperture of the switches), a 15 hours averaging was necessary. The results are in good agreement with quantum mechanics, violating the Bell inequalities by 5 standard deviations.

\footnotetext{
${ }^{7}$ Each analyzer was a pile of plate polarizers.

${ }^{8}$ The polarizers were polarizing cubes, made of two prisms with suitable dielectric thin films on their sides stuck together.
} 


\subsection{Toward the ideal experiment}

After the above work of the Orsay group in the early 80's, one could conclude that all recent experiments confirm the predictions of quantum mechanics and that no additional test is required. However, from a strictly logical point of view, the above experiments do not succeed in ruling out a local realistic explanation completely, because of two essential loopholes: locality and detection.

The locality loophole remains open in the third experiment by Aspect et al. [25] due to the periodic sinusoidal switching used. Thus communication slower than the speed of light, or even at the speed of light, could in principle explain the results obtained. In fact, the condition of locality was fully enforced for the first time only in 1998 by Weihs and co-worker [26] in Innsbrück. In their experiment, the necessary space-like separation of the observations is achieved by:

i) sufficient physical distance between the measurement stations (440 m),

ii) ultra-fast and random setting of the analyzers,

iii) completely independent data registration, the timing being monitored by local rubidium atomic clocks.

This crucial experiment has become possible due to the development of new sources of correlated photons [27]. In these sources, a pair of red photons is produced by a parametric down conversion of a U.V. photon (pump beam) in a non-linear crystal. Due to the phase matching condition (determined by the orientation of the crystal axes relative to the pump beam), there is a strong correlation between the directions of emission of the two photons of a pair (in contrast to the atomic radiative cascades which produce photons only weakly correlated in direction). Consequently, larger coincidence rates can be obtained (more than one order of magnitude larger than in atomic radiative cascade). Moreover, the production of two narrow beams of correlated photons permits one to feed them into two optical fibres. Then, from a practical point of view, with these new schemes:

i) it becomes possible to work with small integrated electro-optical devices,

ii) the detectors can be kilometres apart.

Weihs et al observed a violation of the CHSH inequality of 100 standard deviations! These results close the locality loophole.

It is worth noting that the new parametric down conversion sources present another interesting feature. They produce entangled states with correlation between observables other than polarization, namely time of emission, energy, direction of emissions. Among the amount of experiments performed during the 15 last years, two of them can be mentioned: they present an experimental demonstration of quantum correlations over $4 \mathrm{~km}$ [28] and $10 \mathrm{~km}$ [29] using optical fibres, concluding that the distance does not destroy the entanglement.

The so-called "detection loophole" relies on the fact that so far all experiments detected only a small subset of all pairs created [30]. It is therefore necessary to assume that the pairs registered are a fair sample of all pairs emitted ("fair sampling assumption") as emphasized by J.S. Bell: "... it is hard for me to believe that quantum mechanics works so nicely for inefficient practical set-ups and is yet going to fail badly when sufficient refinements are made". However, experiments have been performed in order to try to close the detection loophole. Due to a too low efficiency of currently available photon detectors, people also used massive particles which are easier to detect. Conclusive tests of the Bell inequalities have been realized by Rowe et al [31]. Two trapped ions are prepared in an entangled state by Raman laser beams. The agreement with quantum mechanics is excellent, making their experiment the first violation of the Bell inequalities with high enough efficiency. However one has to mention that the two ions in the same trap are very close to each other, so that the detection events are not space-like separated and it seems difficult to fulfil the timing conditions in experiments following this scheme.

Entanglement experiments have also been performed with Rydberg atoms and microwave photons in a cavity. However, the signal contrast in these experiments [32] is not high enough to observe a violation of the Bell inequalities. 


\section{Neo-Bohmian interpretation}

\subsection{Orthodox interpretation, a few comments}

In the physics community it is frequently stated that "it is not possible to know simultaneously the position and the momentum of a given particle, since the position and momentum operators are non-commutative". This statement leads to a naive realistic point of view concerning the use of operators in quantum mechanics, as primary "real" objects and not as emerging quantities. Let us say, with Bohr, that what we call "quantum observables" acquire their significance only through their association with specific experiments (measurements). In the Hilbert space parlance, the relevant quantities associated with a given experiment are the partition of subspaces $\mathcal{H}_{\alpha}$ and the values $\lambda_{\alpha}$ associated with the final result of the experiment. The collection $\left\{\mathcal{H}_{\alpha}, \lambda_{\alpha}\right\}$ is compactly represented by the self-adjoint operator

$$
A=\sum_{\alpha} \lambda_{\alpha} P_{\alpha}
$$

where $P_{\alpha}$ is the projector onto the corresponding Hilbert subspace $\mathcal{H}_{\alpha}$. In this way a given experiment $\epsilon$ is associated with a given operator $A_{\epsilon}$ which finally is interpreted as having a reality that is linked to the system alone and independent of the actual reproducible experiment.

\subsection{Bohmian interpretation}

The goals of such an interpretation. The principal goal of the Bohmian interpretation is clear - to restore realism. It is an attempt to give a clear ontology to the constitutive elements of the world $[34,35,37]$. On the most simple level of quantum mechanics, we can state within this interpretation that the fundamental elements are particles which are really particles and consequently they have a perfectly definite position and velocity at all times. This does not mean that such an interpretation is focused on the particle like structure of matter, since a field ontology is also perfectly possible. Once more, the goal is to give a clear ontology. Within the particle picture, the lack of causality/determinizm of quantum mechanics is of the same type as that encountered in classical statistical mechanics, basically due to our lack of knowledge of the initial conditions. But one should not confuse this approach with a return to the classical Newtonian concepts.

de Broglie - Bohmian theory. In 1927, at the Solvay congress, the young French physicist Louis de Broglie proposed a quantum theory known as the pilot-wave theory [34]. His theory is grounded on two hypotheses:

i) there exists a continuous field $\phi=R \mathrm{e}^{\mathrm{i} S / \hbar}$ satisfying the Schrödinger equation and

ii) the necessity that a particle, of mass $m$, follows the trajectories defined by $m \mathbf{v}=\nabla S$.

This is basically the interpretation proposed some thirty years later by David Bohm [35]. The complete description within the pilot-wave interpretation of a system of $N$ particles is specified by the wave function

$$
\Psi(q, t) \quad q=\left(q_{1}, q_{2}, \ldots, q_{N}\right) \in \mathbb{R}^{3 N},
$$

and the coordinates,

$$
Q=\left(Q_{1}, Q_{2}, \ldots, Q_{N}\right) \in \mathbb{R}^{3 N},
$$

of the particle positions. The wave function $\Psi(q, t)$ evolves according to the Schrödinger equation

$$
\mathrm{i} \hbar \frac{\partial}{\partial t} \Psi=H \Psi,
$$

where

$$
H=-\sum_{k} \frac{\hbar^{2}}{2 m_{k}} \nabla_{k}^{2}+V
$$


and pilots the motion of the particles according to the equation

$$
\frac{\mathrm{d} Q_{k}}{\mathrm{~d} t}=\left.\frac{\hbar}{m_{k}} \frac{\operatorname{Im}\left(\Psi^{*} \nabla_{k} \Psi\right)}{\Psi^{*} \Psi}\right|_{Q_{1}, \ldots, Q_{N}} .
$$

Hence, one can try to write a generalized Jacobi equation where, together with the classical potential, a "quantum potential" appears, leading to a modification of Newtonian mechanics via a quantum force. This attempt however is not really suitable since it does not emphasize the profound epistemological change from Newtonian mechanics to quantum mechanics. Putting the wave function $\Psi=R \mathrm{e}^{\mathrm{i} S / \hbar}$ into the Schrödinger equation, the real and imaginary parts lead to two equations. the first of these is the Hamilton-Jacobi equation

$$
\frac{\partial S}{\partial t}+H(\nabla S, q)+U=0
$$

where

$$
U=-\sum_{k} \frac{\hbar^{2}}{2 m_{k}} \frac{\nabla_{k}^{2} R}{R}
$$

is the so-called quantum potential, the only quantity in the Hamilton-Jacobi equation proportional to the Planck constant $[34,35]$. The second equation is a continuity equation,

$$
\frac{\partial \rho}{\partial t}+\operatorname{div}(\rho v)=0
$$

with $v=\left(v_{1}, \ldots, v_{N}\right)$ and div the divergence in configuration space. The particle velocities are given by

$$
v_{k}=\frac{\nabla_{k} S}{m_{k}} .
$$

This rewriting of the quantum equations suggests that the quantum nature of matter lies only on a slight modification of the classical equations, basically by the appearance of the quantum potential. This interpretation is tempting and was indeed adopted in the past. However the de Broglie-Bohm theory should actually be considered as a new theory with its own corpus of concepts [38]. For example, the particle masses are not the coefficients appearing in Newton dynamical law but rather the factors of the field equation and entering into the guiding equations. On the same lines, the velocities in the de Broglie-Bohm theory are not independent of the positions since they are given by the guiding equations. What is fundamental here in this interpretation of the theory is the guidance condition and not the quantum potential which can be viewed at best as a good picture when taking the classical limit of the theory.

From the very beginning, the pilot-wave theory had to face several criticisms. The first is that there is no back reaction of the particles on the field $\Psi$, which is unusual in physics. Another criticism is that the field itself, contrary to a physical field, does not live in the physical space but rather in the much larger configuration space $\mathbb{R}^{3 N}$. This leads to the non-local and non-separable character of the theory. Finally, if one requires that this theory be consistent with the usual quantum theory predictions, one should demand that the positions of the particles at the initial time $t_{0}$ should be distributed according to the law

$$
\rho\left(q, t_{0}\right)=\left|\Psi\left(q, t_{0}\right)\right|^{2},
$$

and they will be distributed at a latter time as

$$
\rho(q, t)=|\Psi(q, t)|^{2}
$$

One of the criticisms was that within this theory, the wave function plays at the same time two apparently irreconciable roles. It determines the quantum potential acting on the particles and defines a probability density associated with the trajectories. Evidently, the most important point is 
to give a strong argument, possibly of the same type as that given in classical statistical mechanics, leading to the quantum distribution of the initial positions [36].

Probabilities. The important point in order to recover and save the usual quantum mechanics power is that the probability distribution for a system, which has an associated wave function $\Psi$, should be given by $\rho(q)=|\Psi(q)|^{2}$ at an initial instant. If this is so, the continuity equation, extracted from the Schrödinger equation, will guaranty that the system will stay $|\Psi(q, t)|^{2}$ distributed at a later time. This condition is called the quantum equilibrium hypothesis [36,37]. Accordingly, on a system in quantum equilibrium it is not possible to go beyond the distribution $|\Psi|^{2}$ and consequently no deviation or violation of the Heisenberg inequalities will be ever observed. In other words, the clear ontology introduced via, for example, the particle picture, will neither remove nor erase the "paradoxical" quantum behaviour of the system, since it is in quantum equilibrium. The gain compared to the orthodox interpretation is that now the indeterminism is empirical rather than ontological.

The remaining point is to address the following question. Why is the system of interest in quantum equilibrium? From the very beginning of the de Broglie-Bohm interpretation, this was the difficult aspect to be clarified. Several attempts were made in several directions, modifying the original theory or trying to set up a dynamical foundation of the quantum equilibrium hypothesis. It is not clear if such an attempt is accessible or feasible. However it could be as well that the equilibrium hypothesis has to be postulated as an empirical fact. More recently, in another direction of thought, Dürr et al. proposed an explanation à la Boltzmann [37]. The essential point is the notion of a partial wave function associated with a subsystem of a much larger system, exactly in analogy with the emergence of the canonical distribution when considering a small part of an isolated total system, the total system being in a "typical" state. Indeed, if one takes seriously enough the de Broglie-Bohm theory one should expect that the behaviour of a subsystem is completely determined by the wave function $\Psi(q)$ of the universe and its corresponding configuration. Dürr et al. associate a wave function with a subsystem within the following decomposition of the universe wave function,

$$
\Psi(x, y)=\psi(x) \Phi(y)+\Psi^{\perp}(x, y)
$$

where $x$ stands for the system variable while $y$ is associated with the rest of the universe. The functions $\Phi$ and $\Psi^{\perp}$ have macroscopically distinct supports for the variable $y$. So, if the configuration variable $Y$ lies into the $\Phi$ support, we have

$$
\Psi(x, Y)=\psi(x) \Phi(Y)
$$

and one is able to associate a wave function with the subsystem. The preceding structure of the total wave function is justified within the decoherence mechanism, i.e. the irreversible flow of the coherent phase into the so-called environment, that is the rest of the universe.

Since, as stated by Mach and others, the universe is given only once, it is meaningless to associate with it a distribution $\rho$ from its wave function $\Psi$. Nevertheless, Dür et al. argued on the base of "time independence" that $|\Psi|^{2}$ gives a measure of typicality and then, within a typical universe that is over an overwhelming majority of possible initial universe configurations, the equilibrium hypothesis holds for the subsystems [37]. Objections to this derivation were formulated since it is not clear at all why dynamical considerations should play any role in order to specify the initial conditions.

The wave function as a law. In their formulation of Bohmian mechanics, Dürr et al. proposed a very interesting interpretation of the wave function as a law governing the dynamics rather than specifying the state of the system [38]. Indeed, if we suppose that the function of the universe is given by a law of the form

$$
H_{U} \Psi=0
$$

the solution $\Psi$ of this equation together with the initial configuration $Q$ determines perfectly the future evolution of the positions, thanks to the guiding equation,

$$
\frac{\mathrm{d}}{\mathrm{d} t} Q=D(\log \Psi)
$$


where $D$ is a differential operator. We see here the analogy with, for example, Hamilton's equations

$$
\frac{\mathrm{d}}{\mathrm{d} t} \xi=D H
$$

and one is tempted to identify the role of $\log \Psi$ with that of the Hamiltonian $H$. In that sense, $\Psi$ plays the role of the law governing the motion of the particles. This interpretation has also the merit of providing an explanation of the "bizarre" fact that the wave function lives in the configuration space and not in the real space as other physical fields.

Finally, within this picture, one may notice that the time-dependent Schrödinger equation for a system should emerge from the universe equation $H_{U} \Psi$ when restricting to the system wave function $\psi$. In sufficiently simple models such a reduction was accomplished explicitly.

\subsection{Criticism of the de Broglie-Bohm theory}

Since the very beginning, what is usually called the de Broglie-Bohm theory or the pilot-wave theory has received much criticism. These include the return to classical concepts, the lack of new predictions compared to the standard quantum mechanics, asymmetry with respect to position and momentum, the introduction of myriads of empty waves, the foundation of the quantum equilibrium hypothesis, as well as its relativistic and quantum field generalizations. This list is certainly not exhaustive but it is intended to be long enough to give to the reader the impression of a not-so-well founded theory. However, most of the criticism could be answered with convincing arguments and none of these objections are rigorous disproof of the de Broglie-Bohm theory. One can refer to Passon [39] for a short reply to all these objections.

\section{Discussion}

Realism is the assumption that there exists an objective external world independent of our perception of it. In a realistic physical theory, one thus requires a clear ontology of the basic "objects" used - for example fields which are really fields, particles which are really particles, etc. $[37,38]$. Locality means that these objects are defined locally with no instantaneous action at a distance. Local realism may thus be defined by the combination of the principle of locality with the assumption that all objects must objectively have their properties already before they are observed. The paradigmatic example is that of local hidden variables.

To summarize the experimental contribution to this debate, one can conclude that, since the pioneering ones in the sixties, an impressive amount of studies are in agreement with quantum mechanics, or at least contradict local hidden-variable theories. Today, even in the absence of an ultimate experiment - where both the detection loophole would be definitely closed and locality enforced - one can conclude there is a failure of local realizm. It would be interesting to improve the detection process in such experiments, as recently proposed by Garcia-Patron et al. [33]. However we do not consider that the detection loophole is as crucial as the locality one.

It is nevertheless important to underline that local hidden-variable theories have been ruled out but that the hidden-variable theories in general have not been disqualified. For example, the Bohmian mechanics or stochastic approaches are not invalidated, since they are non-local theories. Although the neo-Bohmian interpretation offers a promising alternative and gives an example of the possibility of a deterministic interpretation with a clear ontology, it suffers from an essential weakness. This is the overly-close relationship with the usual quantum mechanics, since it was specially designed to restore the predictions made by ordinary quantum mechanics. Consequently, neo-Bohmian and Quantum Mechanics are examples of empirically equivalent theories and it is not clear whether one should expect a significant progress in the interpretation restricting only to empirically equivalent theories. Stochastic approaches open a perspective, but suffer from the difficulty of admiting a generalization in a relativistic context.

Eventually we can summarize the basic lines of the orthodox interpretation as being within the essential probabilizm, forbidding the possibility of an ontological determinizm, and the complementarity principle, stating that it is only possible within one experiment to reach partial aspects 
of reality which are mutually excluded. Consistently with this approach, one may comment that the perfect knowledge of the state of the system leads in general, nevertheless, to probabilistic aspects, that is to intrinsic stochasticity. The truth is not elsewhere, deeper, but on the surface. In some sense, we recover again the idea of an achievement of science, an old idea that seems to frequently accompany the new theories. It is amazing to notice that so much efforts of research and pedagogical type have been made into the direction of impossibility theorems by those who themselves have discovered and built the theory. In other areas of human activity this would be suspicious, in physics it is not.

\section{Acknowledgements}

We acknowledge Malte Henkel for his kind help in the translation of quotations from Einstein. We also would like to express our gratitude to Ralph Kenna for his careful reading of the mansucript, and more precisely for his rewriting many sentences, trying to make a "frenglish" text almost british, or at least readable by english-speaking persons.

\section{References}

1. Genovese M., Research on hidden variable theories: A review of recent progresses. Phys. Rep., 2005, 413, 319 .

2. Einstein A., Podolsky B., Rosen N., Can quantum-mechanical description of physical reality be considered complete? Phys. Rev., 1935, 47, 777.

3. Bohm D. Quantum theory. Dover, New-York, 1989, 611-623.

4. Bohr N., Can quantum-mechanical description of physical reality be considered complete? Phys. Rev., 1935, 48, 696.

5. For further details concerning Bohr's interpretation, see Bohr. N. La théorie atomique et la description des phénomènes. Gauthier-Villars, Paris 1932 (new publishing Ed. Jacques Gabay, 1993). See also Bohr N. Physique atomique et connaissance humaine. Gauthier-Villars, Paris 1961 (new publishing Ed. Gallimard 1991).

6. Einstein A. Albert Einstein, (Euvres choisies, 1 : Quanta. Ed. by F. Balibar, Seuil, CNRS, Paris, 1989

7. Einstein A., Mécanique quantique et réalité Dialectica, 1948, II, 320., trad. Fr. in Einstein A. Albert Einstein, Euvres choisies, 1 : Quanta. Ed. by F. Balibar, Seuil, CNRS, Paris 1989, 244-249.

8. von Neumann J. Mathematische Grundlagen der Quantenmechanik. Springer, Berlin, 1932.

9. Hemmick D. Hidden Variables and Nonlocality in Quantum Mechanics. Dissertation, Rutgers Univ. 1996, quant-ph/0412011.

10. Schrödinger E., Die gegenwärtige Situation in der Quantenmechanik. Die Naturwissenschaften, 1935, 23, 807, 823, 844 .

11. Gleason A.M., Measures on the closed subspaces of a hilbert space. J. Math. and Mech., 1957, 6, 885.

12. Kochen S., Specker E.P., The problem of hidden variables in quantum mechanics. J. Math. and Mech., 1967, 17, 59 .

13. Bell J.S., On the Einstein-Podolsky-Rosen Paradox. Physics, 1964, 1, 195.

14. Clauser J.F., Horne M.A., Shimony A., Holt R.A., Proposed experiment to test local hidden-variable theories. Phys. Rev. Lett., 1969, 23, 880.

15. Bohm D., Aharonov Y., Discussion of Experimental Proof for the Paradox of Einstein, Rosen and Podolsky. Phys. Rev., 1957, 108, 1070.

16. Aspect A. Ph.D. thesis University Paris-Sud, France. Trois tests expérimentaux des inégalités de Bell par mesure de corrélation de polarisation de photons, 1983.

17. Foundations of quantum mechanics, B. D'Espagnat ed., Proc. of the 49th Varenna School, Academic, New-York, 1971.

18. Aspect A., Bell's inequality test: more ideal than ever. Nature, 1999, 398, 189.

19. Freedman S.J., Clauser J.F., Experimental test of local hidden-variable theories. Phys. Rev. Lett., 1972, 28, 938.

20. Pipkin F.M. Atomic Physics Tests of the Basics Concepts in Quantum Mechanics. Advances in Atomic and Molecular Physics, ed. by D.R. Bates, B. Bederson. Academic, London, 1978.

21. Clauser J.F., Experimental investigation of a polarization correlation anomaly. Phys. Rev. Lett., 1976, 36, 1223. 
22. Fry E.S., Thompson R.C., Experimental test of local hidden-variable theories. Phys. Rev. Lett., 1976, 37, 465.

23. Aspect A., Grangier P., Roger G., Experimental tests of realistic local theories via Bell's theorem. Phys. Rev. Lett., 1981, 47, 460.

24. Aspect A., Grangier P., Roger G., Experimental realization of Einstein-Podolsky-Rosen-Bohm gedankenexperiment: a new violation of Bell's inequalities. Phys. Rev. Lett., 1982, 49, 91.

25. Aspect A., Dalibard J., Roger G., Experimental test of Bell's inequalities using variable analyzers. Phys. Rev. Lett., 1982, 49, 1804.

26. Weihs G., Jennewein T., Simon C., Weinfurter H., Zeilinger A., Violation of Bell's inequality under strict Einstein locality condition. Phys. Rev. Lett., 1998, 81, 5039.

27. Kwiat P.G., Mattle K., Weinfurter H., Zeilinger A., New High-Intensity Source of PolarizationEntangled Photon-Pairs. Phys. Rev. Lett., 1995, 75, 4337.

28. Tapster P.R., Rarity J.G., Owens P.C.M., Violation of Bell's Inequality over $4 \mathrm{~km}$ of Optical Fiber. Phys. Rev. Lett., 1994, 73, 1923.

29. Tittel W., Brendel J., Zbinden H., Gisin N., Violation of Bell inequalities by photons more than $10 \mathrm{~km}$ apart. Phys. Rev. Lett., 1998, 81, 3563.

30. Grangier P., Quantum physics: Count them all. Nature, 2001, 409, 774.

31. Rowe M.A., Kielpinsky D., Meyer V., Sackett C.A. , Itano W.M., Wineland D.J., Experimental violation of a Bell's inequality with efficient detection. Nature, 2001, 409, 791.

32. Raimond J.M., Brune M., Haroche S., Manipulating quantum entanglement with atoms and photons in a cavity. Rev. Mod. Phys., 2001, 73, 565.

33. Garcia-Patrón R., Fiurášek J., Cerf N.J., Wenger J., Tualle-Brouri R., Grangier Ph., Proposal for a loophole-free Bell test using homodyne detection. Phys. Rev. Lett., 2004, 93, 130409.

34. de Broglie L. La nouvelle dynamique des quanta. Electrons et photons : Rapports et discussions du cinquième conseil de Physique tenu à Bruxelles du 24 au 29 octobre 1927 sous les auspices de l'institut international de physique Solvay, ed. by Gauthier-Villars. Paris, 1928, 105.

35. Bohm D., , A suggested interpretation of the quantum theory in terms of "hidden variables". Phys. Rev., 1952, 85, 166I, 180II.

36. Bohm D., Proof that probability density approaches $|\psi|^{2}$ in causal interpretation of quantum theory. Phys. Rev., 1953, 89, 458.

37. Dürr D., Goldstein S., Zanghi N., Quantum equilibrium and the origin of absolute uncertainty. J. Stat. Phys., 1992, 67, 843.

38. Dürr D., Goldstein S., Zanghi N., Bohmian Mechanics and the Meaning of the Wave Function. Experimental Metaphysics - Quantum Mechanical Studies in Honor of Abner Shimony, ed. R.S.Cohen, M.Horne, J.Stachel. Boston Studies in the Philosophy of Science, Kluwer, 1997.

39. Passon O., Why isn't every physicist a Bohmian? (quant-ph/0412119).

\section{Questions and answers}

$\mathcal{Q}$ (Ara Apkarian): Could you comment on the conceptual distinction between locality and separability.

$\mathcal{A}$ One may define separability in the following way: a separable state is one that can be created from another state by local means, which is not true for an entangled state. Mathematically, one expresses that in the form

$$
\rho=\sum_{k} p_{k} \rho_{A}^{k} \otimes \rho_{B}^{k}, \quad \sum_{k} p_{k}=1,
$$

where $\rho$ is the density matrix of the quantum state and where $\rho_{A, B}$ are density matrices belonging to the Hilbert spaces $\mathcal{H}_{A, B}$. It seems then that locality plays a significant role in the concept of separability. However, if one takes as a definition for locality the fact that distant objects cannot effect each other directly, one implicitly refers to objects, meaning real objects. But, in the so-called orthodox interpretation, the wave function has no direct physical interpretation or reality, meaning that it is the concept of local object that is rejected in general. In a non-separable state, one cannot a priori define local real objects.

$\mathcal{Q}$ (Eduardo Ludeña): Could you comment on the effect of distance on entanglement. 
$\mathcal{A}$ What is important, rather than distance, is the interaction with environment. As long as this interaction remains negligible, the entangled character of a quantum state is preserved.

$\mathcal{Q}$ (Bertrand Berche): This is a personal comment more than a question. I believe that physicits' common conception of a physical theory is very constraining. Couldn't we imagine as admissible a theory which would not be able at all to answer (I mean even not in probabilistic terms) some questions which are a priori from its domain of applicability? Something a bit like Gödel's theorem and propositions which are neither true nor false. Why do we ask so much to our theories? Isn't it due to our custom that physical theories have been so powerful in the past, with the "The Unreasonable Effectiveness of Mathematics in the Natural Sciences" of Eugene Wigner.

$\mathcal{Q}$ (Yurij Holovatch): Is the Bohm theory the only known non-local hidden-variable theory?

$\mathcal{A}$ No, an alternative theory called stochastic mechanics was also pretty much studied. In 1966 , Nelson showed that the Schrödinger equation could be derived from the hydrodynamical equation of a classical fluid made of particles with Brownian trajectories (E. Nelson, Derivation of the Schroedinger Equation from Newtonian Mechanics Phys. Rev., 1966, 150, 1079.). He suggested that the interaction of the particles with the vacuum for instance could induce fluctuations of their position. Because of these fluctuations, the trajectories are continuous but not differentiable. At a given time, two different velocities can be defined: the advanced and the retarded velocities related to each other by reversing the direction of time:

$$
\mathbf{b}(t)=\lim _{\Delta t \rightarrow 0^{+}} \frac{\langle\mathbf{r}(t+\Delta t)-\mathbf{r}(t)\rangle}{\Delta t}, \quad \mathbf{b}_{*}(t)=\lim _{\Delta t \rightarrow 0^{+}} \frac{\langle\mathbf{r}(t)-\mathbf{r}(t-\Delta t)\rangle}{\Delta t},
$$

where $\langle\cdots\rangle$ denotes the average over the fluctuations. At the macroscopic level, the density of particles satisfies a diffusion equation with a diffusion constant depending on the Planck constant. By reversing the direction of time, the sign of this diffusion constant changes. Nelson combined these two equations to get a Navier-Stockes-like hydrodynamical equation

$$
\frac{\hbar}{2 m} \Delta \mathbf{u}-(\mathbf{u} . \nabla) \mathbf{u}+(\mathbf{v} . \nabla) \mathbf{v}+\frac{1}{m} \nabla V=-\frac{\partial \mathbf{v}}{\partial t}
$$

where

$$
\mathbf{v}=\frac{1}{2}\left(\mathbf{b}+\mathbf{b}_{*}\right), \quad \mathbf{u}=\frac{1}{2}\left(\mathbf{b}-\mathbf{b}_{*}\right)
$$

that can then easily be related to the Schrödinger equation with the wave function

$$
\psi=\mathrm{e}^{R+\mathrm{i} S}, \quad \mathbf{v}=\frac{\hbar}{m} \nabla S, \quad \mathbf{u}=\frac{\hbar}{m} \nabla R .
$$

The stochastic mechanics still suffers from several problems. First of all, the interpretation of the theory is not trivial even though some progress have been made by Fritsche and Haugk in 2003 (L. Fritsche et M. Haugk, A new look at the derivation of the Schrodinger equation from Newtonian mechanics Ann. Phys. (Leipzig), 2003, 12, 371.). Moreover, there is still no relativistic generalization of the Nelson approach. 


\title{
Історичні та інтеграційні аспекти квантової механіки: наївний
} підхід фізика

\author{
Б.Берш, К.Шатлен, К.Дюфур, Т.Гурйо, Д.Каревскі \\ Група М, Лабораторія фізики матеріалів, UMR CNRS 7556, \\ Університет Анрі Пуанкаре, Нансі 1, 54506 Вандувр лє Нансі Седекс, Франція \\ Отримано 10 квітня 2006 р., в остаточному вигляді - 17 квітня 2006 р.
}

\begin{abstract}
Протягом останніх 80 років багато теоретичних передбачень квантової механіки було підтверджено експериментально. Однак інтерпретаційні аспекти обговорюються вже тривалий час. Зокрема, все ще відкритими залишаються запитання про існування прихованих змінних. Ми робимо огляд цих проблем, приділяючи особливу увагу їх історичним аспектам. Базуючись на сучасних експериментальних даних, ми приводимо аргументи, що виключають локальний реалізм. Тим не менше, не виключаються інші інтерпретації квантової механіки.
\end{abstract}

Ключові слова: основи квантової механіки, ЕПР, заплутаність, приховані змінні, нерівності Белла, теорія Бома

PACS: $01.65 .+g, 01.70 .+w, 03.65 . T a$ 
